\title{
Perturbation-Based Spectral Stochastic Meshless Local Petrov-Galerkin Method in Predicting Probabilistic Settlements
}

\author{
Guang Yih Sheu*, ${ }^{*}$
}

Department of Accounting Information System, Chang-Jung Christian University, Kway-Jen, Tainan 714, Taiwan

\begin{abstract}
In an attempt of solving stochastic boundary-value problems sufficiently accurately without creating a finite element discretization, a previous study (Comput. Geotech. 2011, vol. 38, No. 4, pp. 407-415) developed the spectral stochastic meshless local Petrov-Galerkin (SSMLPG) method. Some different approaches of deriving an SSMLPG formulation have been developed using various random field discretization methods. This study presents the SSMLPG formulation composed of perturbation expansions of random fields and a 2D meshfreee weak-strong (MWS) form in elasticity. A performance evaluation of this SSMLPG formulation is implemented through a stochastic elastostatic problem in which probabilistic settlements are predicted with the uncertainty in the spatial variability of Young`s modulus. The evaluation results demonstrate that SSMLPG-based predicted probabilistic settlements approach more close to the Monte Carlo simulation (MCS) results than spectral stochastic finite element-based predicted probabilistic settlements do. In addition, generating the SSMLPG results is time-saving than completing the MCS does. In conclusion, the SSMLPG method can be an efficient alternative tool to solve stochastic boundary-value problems.
\end{abstract}

Keywords: Spectral stochastic meshless local Petrov-Galerkin method, perturbation expansion, meshfree weak-strong formulation, uncertainty.

\section{INTRODUCTION}

Solving computational mechanic problems may encounter the uncertainties contributed by such as the spatial variability of material properties and complex scenarios of imposed loadings. Accounting for such uncertainties is necessary, since these uncertainties can cause unreliable numerical results for the design purpose.

One of the approaches for solving computational mechanic problems with accounting for the possible uncertainties is assuming random fields to represent those uncertainties and regarding the corresponding computational mechanic problem as a stochastic boundary-value problem. To solve a stochastic boundary-value problem, we can apply the spectral stochastic finite element (SSFEM) method [1]. Briefly, deriving an SSFEM formulation couples a conventional finite element formulation with the representations of random fields. These representations of random fields can be derived by such as the perturbation and Karhunen-Loève expansions.

A number of SSFEM formulations are available for some branches of engineering. References [2-3] are two recent examples. However, some other studies; for example, Ref. [4], reported deficiencies, such as mesh distortion under

*Address correspondence to this author at the Department of Accounting Information System, Chang Jung Christian University, No. 396, Sec. 1, Chang-Jung Rd., Kway Jen, Tainan, Taiwan; Tel: 886-6-2785123-2225; Fax: 886-6-2785683;

E-mails: xsheu@hotmail.com,xsheu@mail.cjcu.edu.tw

${ }^{\S}$ Also Adjunct Associate Professor, Department of Civil Engineering, FengChia University, Taichung, Taiwan. large deformations or re-meshing around the crack tip in the crack propagation, in applying the SSFEM method. To eliminate these deficiencies thereby improve the computational efficiency, a previous study [5] extended the meshless local Petrov-Galerkin (MLPG) method [6] to the SSMLPG method. Applying the SSMLPG method does not need a finite element discretization. Therefore, the time spent to create a finite element discretization or background cells for the numerical integration can be saved. Nonetheless, the SSMLPG results of two elastostatic problems approach more satisfactorily to the MCS results than the SSFEM results of the same problems do [5].

Some different approaches of deriving the SSMLPG formulation have been developed using various random field discretization methods. The succeeding study presents the SSMLPG formulation containing perturbation expansions of random fields and the 2D MWS form in elasticity [7]. In addition, the radial basis function (RBF) is used to construct the meshfree shape function. A performance evaluation of this perturbation-based SSMLPG formulation is implemented through a stochastic elastostatic problem in which probabilistic settlements are predicted subjected to the uncertainty in the spatial variability of Young 's modulus.

The remainder of this study is organized into 4 sections. In Sec. 2, deriving a 2D MWS form in elasticity is reviewed. In Sec. 3, equating the perturbation expansion of Young`s modulus and deducing the perturbation-based SSMLPG formulation is presented. In Sec. 4, a performance evaluation of resulting SSMLPG formulation is implemented. Discussing the evaluation results to draw some conclusion is presented in Sec. 5. 


\section{TWO-DIMENSIONAL MESHFREE WEAK- STRONG FORMULATION IN ELASTICITY}

Assume linearly elastic and isotropic material and the infinitesimal strain assumption holds. Suppose $\Omega$ is a problem domain, $\mathbf{x}=\left(\mathrm{x}_{1}, \mathrm{x}_{2}\right)$ is a vector of spatial coordinates, and $\theta$ is an event in the probability space. Describe each physical parameter within $\Omega$ as functions of $\mathbf{x}$ and $\theta$. This study derives the 2D MWS form in elasticity by the following differential equation:

$\sigma_{i j, j}+b_{i}=0$

where $\sigma_{i j}$ are the stress fields corresponding to the displacement fields $\mathrm{u}_{\mathrm{i}}, \mathrm{b}_{\mathrm{i}}$ are the body forces, and $(\cdot)_{, j}=\frac{\partial(\cdot)}{\partial x_{j}}$. The boundary conditions are given by

$t_{i}=\sigma_{i j} n_{j}=\bar{t}_{i} \quad$ on the natural boundary $\Gamma_{\mathrm{T}}$

$u=\bar{u}_{i} \quad$ on the essential boundary $\Gamma_{\mathrm{U}}$

where the overbar represents the prescribed data, $t_{i}$ are the tractions, $\mathrm{n}_{\mathrm{j}}$ are the components of a unit vector $\mathbf{n}$ outward normal to $\Gamma$, and $\Gamma=\Gamma_{\mathrm{U}} \cup \Gamma_{\mathrm{T}}$.

\section{Meshfree Strong Form}

Suppose there are $\mathrm{N}_{\mathrm{T}}$ nodes within $\Omega$. In addition, $\Omega_{\mathrm{S}}$ is a local quadrature domain for a node $\mathbf{x}_{\mathrm{I}}\left(\mathrm{I}=1\right.$ to $\left.\mathrm{N}_{\mathrm{T}}\right)$ and $\Gamma_{\mathrm{S}}$ is its boundary. If $\Omega_{\mathrm{S}}$ for a node $\mathbf{x}_{\mathrm{I}}$ does not interact with $\Gamma_{\mathrm{T}}$, a meshfree strong form of Eq. (1) is applied at this node. We can derive this meshfree strong form by first simplifying $\sigma_{\mathrm{ij}}$ by specific stress-strain and strain-displacement relationships. The resulting expressions are next substituted into Eq. (1). For simplicity, the succeeding study focuses on the plain strain condition; therefore, $\sigma_{i j}$ are simplified by

$$
\begin{aligned}
\left\{\begin{array}{c}
\sigma_{11} \\
\sigma_{22} \\
\sigma_{12}
\end{array}\right\} & =\frac{E(1-v)}{(1+v)(1-2 v)}\left[\begin{array}{ccc}
1 & \frac{v}{1-v} & 0 \\
\frac{v}{1-v} & 1 & 0 \\
0 & 0 & \frac{1-2 v}{2(1-v)}
\end{array}\right]\left\{\begin{array}{c}
\varepsilon_{11} \\
\varepsilon_{22} \\
\varepsilon_{12}
\end{array}\right\} \\
& =\frac{E(1-v)}{(1+v)(1-2 v)}\left[\begin{array}{ccc}
1 & \frac{v}{1-v} & 0 \\
\frac{v}{1-v} & 1 & 0 \\
0 & 0 & \frac{1-2 v}{2(1-v)}
\end{array}\right]\left[\begin{array}{cc}
\frac{\partial}{\partial x_{1}} & 0 \\
0 & \frac{\partial}{\partial x_{2}} \\
\frac{\partial}{\partial x_{2}} & \frac{\partial}{\partial x_{1}}
\end{array}\right]\left\{\begin{array}{l}
u_{1} \\
u_{2}
\end{array}\right\}
\end{aligned}
$$

where $\varepsilon_{\mathrm{ij}}(\mathrm{i}, \mathrm{j}=1$ to 2$)$ is the strain field. Substituting Eq. (3) into Eq. (1) results in

$$
\begin{aligned}
& \frac{E(1-v)}{(1+v)(1-2 v)}\left[\frac{\partial^{2} u_{1}}{\partial x_{1}^{2}}+\frac{1-2 v}{2(1-v)} \frac{\partial^{2} u_{1}}{\partial x_{2}^{2}}+\frac{1}{2(1-2 v)} \frac{\partial^{2} u_{2}}{\partial x_{1} \partial x_{2}}\right]+b_{1}=0 \\
& \frac{E(1-v)}{(1+v)(1-2 v)}\left[\frac{\partial^{2} u_{2}}{\partial x_{2}^{2}}+\frac{1-2 v}{2(1-v)} \frac{\partial^{2} u_{2}}{\partial x_{1}^{2}}+\frac{1}{2(1-2 v)} \frac{\partial^{2} u_{1}}{\partial x_{1} \partial x_{2}}\right]+b_{2}=0
\end{aligned}
$$

\section{Local Weak Form}

If $\Omega_{\mathrm{S}}$ for a node $\mathbf{x}_{\mathrm{I}}\left(\mathrm{I}=1\right.$ to $\left.\mathrm{N}_{\mathrm{T}}\right)$ interacts with $\Gamma_{\mathrm{T}}$, a local weak form of Eq. (1) is applied at this node. Deducing this local weak form initiates from the following equation:

$\int_{\Omega_{S}}\left(\sigma_{i j, j}+b_{i}\right) \beta_{I} d \Omega=0$

where $\beta_{I}\left(I=1\right.$ to $\left.N_{T}\right)$ is the test function associated with $\mathbf{x}_{I}$. The succeeding study intends to construct the meshfree shape function $\phi$ by the RBF; therefore, the resulting $\phi$ satisfies the Kronecker delta function property $\left(\phi_{\mathrm{IJ}}=0\right.$ for $\mathrm{I} \neq$ $\mathrm{J}, \phi_{\mathrm{IJ}}=0$ for $\mathrm{I}=\mathrm{J}$, and $\mathrm{I}, \mathrm{J}$ denote two nodes). Consequently, neither Lagrangian multipliers nor penalty parameters are required in Eq. (5) for imposing the essential boundary condition. Meanwhile, simplifying Eq. (5) by the divergence theorem yields

$$
\begin{aligned}
& \int_{\Omega_{S}} \sigma_{i j} \beta_{I, j} d \Omega-\oint_{L_{S}} \beta_{I} t_{i} d \Gamma-\oint_{\Gamma_{S U}} \beta_{I} t_{i} d \Gamma \\
& =\oint_{\Gamma_{S T}} \beta_{I} \bar{t}_{i} d \Gamma+\int_{\Omega_{S}} b_{i} \beta_{I} d \Omega
\end{aligned}
$$

where $\Gamma_{\mathrm{ST}}=\Omega_{\mathrm{S}} \cap \Gamma_{\mathrm{T}}, \Gamma_{\mathrm{SU}}=\Omega_{\mathrm{S}} \cap \Gamma_{\mathrm{U}}$, and $\mathrm{L}_{\mathrm{S}}=\Gamma_{\mathrm{S}}-\Gamma_{\mathrm{ST}}-\Gamma_{\mathrm{SU}}$. Theoretically speaking, the shape of $\Omega_{\mathrm{S}}$ can be arbitrary in integrating Eq. (6). However, $\Omega_{\mathrm{S}}$ can be a rectangle centered at $\mathbf{x}_{\mathrm{I}}\left(\mathrm{I}=1\right.$ to $\left.\mathrm{N}_{\mathrm{T}}\right)$ for integrating Eq. (6) more easily. In addition, $\Omega_{\mathrm{s}}$ for $\mathbf{x}_{\mathrm{I}}$ may be different from $\Omega_{\mathrm{Q}}$ for the same node. Fig. (1) outlines the difference between $\Omega_{\mathrm{S}}$ and $\Omega_{\mathrm{Q}}$. Moreover, interpolating the distribution of an unknown or a random field can be based on different interpolation domains or points.

Next, $\beta_{\mathrm{I}}\left(\mathrm{I}=1\right.$ to $\left.\mathrm{N}_{\mathrm{T}}\right)$ is set by

$\beta_{I}(\mathbf{x})= \begin{cases}R_{I}\left(r_{I}\right) & \mathbf{x} \in \Omega_{\mathrm{s}} \\ 0 & \text { otherwise }\end{cases}$

Substituting Eq. (7) into Eq. (6) leads to

$$
\begin{array}{r}
\int_{\Omega_{S}} \sigma_{i j}\left[R_{I}\left(\mathrm{r}_{\mathrm{I}}\right)\right]_{\mathrm{j}} d \Omega-\oint_{L_{S}}\left[R_{I}\left(\mathrm{r}_{\mathrm{I}}\right)\right] t_{i} d \Gamma-\oint_{\Gamma_{S U}}\left[R_{I}\left(\mathrm{r}_{\mathrm{I}}\right)\right] t_{i} d \Gamma \\
=\oint_{\Gamma_{S T}}\left[R_{I}\left(\mathrm{r}_{\mathrm{I}}\right)\right] \overline{t_{i}} d \Gamma+\int_{\Omega_{S}} b_{i}\left[R_{I}\left(\mathrm{r}_{\mathrm{I}}\right)\right] d \Omega
\end{array}
$$

Furthermore, $t_{i}(i=1,2)$ are simplified by Eqs. (2a) and (3). Thus

$$
\begin{aligned}
\left\{\begin{array}{l}
t_{1} \\
t_{2}
\end{array}\right\} & =\left[\begin{array}{lll}
n_{1} & 0 & n_{2} \\
0 & n_{2} & n_{1}
\end{array}\right]\left\{\begin{array}{c}
\sigma_{11} \\
\sigma_{22} \\
\sigma_{12}
\end{array}\right\} \\
& =\left[\begin{array}{ccc}
n_{1} & 0 & n_{2} \\
0 & n_{2} & n_{1}
\end{array}\right] \frac{E(1-v)}{1+v(1-2 v)}\left[\begin{array}{ccc}
1 & \frac{v}{1-v} & 0 \\
\frac{v}{1-v} & 1 & 0 \\
0 & 0 & \frac{1-2 v}{2(1-v)}
\end{array}\right]\left[\begin{array}{cc}
\frac{\partial}{\partial x_{1}} & 0 \\
0 & \frac{\partial}{\partial x_{2}} \\
\frac{\partial}{\partial x_{2}} & \frac{\partial}{\partial x_{1}}
\end{array}\right]\left\{\begin{array}{c}
u_{1} \\
u_{2}
\end{array}\right\}
\end{aligned}
$$

Substituting Eq. (9) into Eq. (8) yields

$$
\begin{aligned}
& \int_{\Omega_{S}} \mathbf{V}_{I}^{T} \mathbf{D B u} d \Omega-\oint_{L_{S}} \mathbf{W}_{I} \mathbf{n D B u} d \Gamma-\oint_{\Gamma_{S U}} \mathbf{W}_{I} \mathbf{n D B u} d \Gamma \\
& =\oint_{\Gamma_{S T}} \mathbf{W}_{I} \overline{\mathbf{t}} d \Gamma+\int_{\Omega_{S}} \mathbf{W}_{I} \mathbf{b} d \Omega
\end{aligned}
$$

where $\mathbf{u}=\left[\mathrm{u}_{1}, \mathrm{u}_{2}\right]^{\mathrm{T}}, \overline{\mathbf{t}}=\left[\bar{t}_{1}, \bar{t}_{2}\right]^{T}, \mathbf{b}=\left[\mathrm{b}_{1}, \mathrm{~b}_{2}\right]^{\mathrm{T}}$, and 


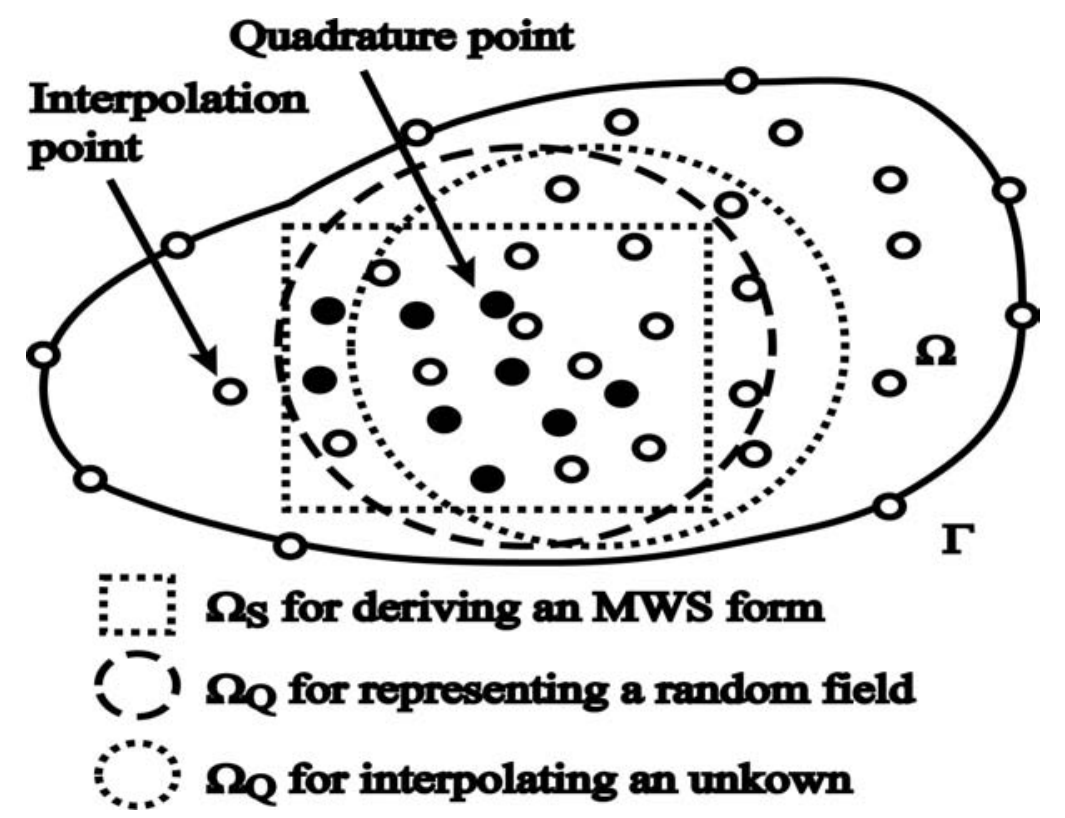

Fig. (1). Difference between $\Omega_{\mathrm{S}}$ and $\Omega_{\mathrm{Q}}$.

$\mathbf{n}=\left[\begin{array}{ccc}n_{1} & 0 & n_{2} \\ 0 & n_{2} & n_{1}\end{array}\right], \quad \mathbf{D}=\frac{E(1-v)}{(1+v)(1-2 v)}\left[\begin{array}{ccc}1 & \frac{v}{1-v} & 0 \\ \frac{v}{1-v} & 1 & 0 \\ 0 & 0 & \frac{1-2 v}{2(1-v)}\end{array}\right]$,

$\mathbf{B}=\left[\begin{array}{cc}\frac{\partial}{\partial x_{1}} & 0 \\ 0 & \frac{\partial}{\partial x_{2}} \\ \frac{\partial}{\partial x_{2}} & \frac{\partial}{\partial x_{1}}\end{array}\right], \mathbf{V}_{I}=\left\{\begin{array}{cc}{\left[R_{I}\left(r_{I}\right)\right]_{, 1}} & 0 \\ 0 & {\left[R_{I}\left(r_{I}\right)\right]_{, 2}} \\ {\left[R_{I}\left(r_{I}\right)\right]_{, 2}} & {\left[R_{I}\left(r_{I}\right)\right]_{, 1}}\end{array}\right\}$,

$\mathbf{W}_{I}=\left[\begin{array}{cc}R_{I}\left(r_{I}\right) & 0 \\ 0 & R_{I}\left(r_{I}\right)\end{array}\right]$

\section{Discrete Equations}

Similarly manipulating the published RBF interpolation formula [5], we can approximate $u_{i}(i=1,2)$ over $\Omega_{Q}$ for $\mathbf{x}_{I}$ $\left(\mathrm{I}=1\right.$ to $\left.\mathrm{N}_{\mathrm{T}}\right)$ by

$u_{i}(\mathbf{x}, \theta)=\boldsymbol{\Phi}(\mathbf{x}) \mathbf{U}=\sum_{j=1}^{N} \phi_{j}(\mathbf{x}) u_{i, j}(\mathbf{x}, \theta)$

where $\mathrm{N}$ is the total number of nodes within $\Omega_{\mathrm{Q}}, \boldsymbol{\Phi}(\mathbf{x})=$ $\left[\phi_{1}(\mathbf{x}), \phi_{2}(\mathbf{x}) \ldots \phi_{\mathrm{N}}(\mathbf{x})\right], \mathbf{U}=\left[\mathrm{u}_{\mathrm{i}, 1}\left(\mathbf{x}_{1}, \theta\right), \mathrm{u}_{\mathrm{i}, 2}\left(\mathbf{x}_{2}, \theta\right) \ldots \mathrm{u}_{\mathrm{i}, \mathrm{N}}\left(\mathbf{x}_{\mathrm{N}}, \theta\right)\right]^{\mathrm{T}}$. Construction of $\phi$ for further details can be seen in Ref. [5]. Based on Eq. (12), Eqs. (4) and (10) can be re-written more succinctly in matrix algebra as

$\mathbf{K}_{I} \mathbf{u}_{I}=\mathbf{F}_{I}$

where $\mathbf{u}_{\mathrm{I}}=\left[\mathbf{u}_{1}, \mathbf{u}_{2} \ldots . \mathbf{u}_{N}\right]_{I}^{T}$ and $\mathbf{K}_{\mathrm{I}}$ and $\mathbf{F}_{\mathrm{I}}$ are the stiffness and force matrices for $\mathbf{x}_{\mathrm{I}}\left(\mathrm{I}=1\right.$ to $\left.\mathrm{N}_{\mathrm{T}}\right)$. $\mathbf{K}_{\mathrm{I}}$ and $\mathbf{F}_{\mathrm{I}}$ are derived by

$$
\left\{\begin{array}{l}
\mathbf{K}_{I}=\mathbf{B}^{T}(\Phi) \mathbf{D}(\mathbf{x}) \mathbf{B}(\Phi) \\
\mathbf{F}_{I}=-\mathbf{b}(\mathbf{x}) \quad\left(\Omega_{\mathrm{Q}} \cap \Gamma_{\mathrm{T}}=\varnothing\right)
\end{array}\right.
$$

$$
\left\{\begin{aligned}
\mathbf{K}_{I}= & \int_{\Omega_{S}} \mathbf{V}_{I}^{T} \mathbf{D B}(\Phi) d \Omega-\oint_{L_{S}} \mathbf{W}_{I} \mathbf{n D B}(\Phi) d \Gamma \\
& -\oint_{\Gamma_{S U}} \mathbf{W}_{I} \mathbf{n D B}(\Phi) d \Gamma \\
\mathbf{F}_{I}= & \oint_{\Gamma_{S T}} \mathbf{W}_{I} \overline{\mathbf{t}} d \Gamma+\int_{\Omega_{S}} \mathbf{W}_{I} \mathbf{b} d \Omega
\end{aligned}\left(\Omega_{\mathrm{Q}} \cap \Gamma_{\mathrm{T}} \neq \varnothing\right)\right.
$$

$$
\text { in which } \mathbf{B}(\Phi)=\left[\begin{array}{cc}
\frac{\partial(\Phi)}{\partial x_{1}} & 0 \\
0 & \frac{\partial(\Phi)}{\partial x_{2}} \\
\frac{\partial(\Phi)}{\partial x_{2}} & \frac{\partial(\Phi)}{\partial x_{1}}
\end{array}\right]
$$

Repeat the derivation of Eq. (13) for all $\mathrm{N}_{\mathrm{T}}$ nodes and assemble all the resulting equations according to the global numbering system result in

$$
\mathbf{K}_{\left(2 N_{T} \times 2 N_{T}\right)} \mathbf{u}_{\left(2 N_{T} \times 1\right)}=\mathbf{F}_{\left(2 N_{T} \times 1\right)}
$$

Eq. (15) is not ready for use, since the uncertainty in the spatial variability of $\mathrm{E}$ has not been treated. In the next section, representing the spatial variability of $\mathrm{E}$ by the perturbation method is presented.

\section{THE PERTURBATION METHOD}

Theoretical backgrounds of the perturbation method can be consulted with Ref. [8]. Interested readers may refer to this reference.

The first step of deriving the perturbation expansion of $\mathrm{E}$ is simulating $\mathrm{E}$ as a random field and equating Taylor expansions of $\mathbf{K}$ and $\mathbf{u}$ in terms of fluctuations of $\mathrm{E}$.

Suppose $E_{1}, E_{2} \ldots E_{N_{T}}$ are the nodal values of $\mathrm{E}$ and small fluctuations $\Delta E_{j}=E_{j}-\mu_{E}\left(j=1\right.$ to $\left.N_{T}\right)$ in which $\mu_{E}$ is the mean value of $E$. The Taylor expansions of $\mathbf{K}$ and $\mathbf{u}$ at $\Delta \mathrm{E}_{\mathrm{j}}=$ $0\left(\mathrm{j}=1\right.$ to $\left.\mathrm{N}_{\mathrm{T}}\right)$ are given by 


$$
\begin{gathered}
\mathbf{K}=\mathbf{K}_{0}+\sum_{i=1}^{N_{T}} \mathbf{K}_{, i} \Delta E_{i}+\frac{1}{2} \sum_{i=1}^{N_{T}} \sum_{j=1}^{N_{T}} \mathbf{K}_{, i j} \Delta E_{i} \Delta E_{j}+\cdots \\
\mathbf{u}=\mathbf{u}_{0}+\sum_{i=1}^{N_{T}} \mathbf{u}_{, i} \Delta E_{i}+\frac{1}{2} \sum_{i=1}^{N_{T}} \sum_{j=1}^{N_{T}} \mathbf{u}_{, i j} \Delta E_{i} \Delta E_{j}+\cdots
\end{gathered}
$$

where

$$
\begin{array}{lll}
\mathbf{K}_{0}=\mathbf{K}\left(E=\mu_{E}\right), & \mathbf{K}_{, i}=\frac{\partial \mathbf{K}}{\partial E_{i}}\left(E=\mu_{E}\right), & \mathbf{K}_{, i j}=\frac{\partial^{2} \mathbf{K}}{\partial E_{i} \partial E_{j}}\left(E=\mu_{E}\right) \\
\mathbf{u}_{0}=\mathbf{u}\left(E=\mu_{E}\right), & \mathbf{u}_{, i}=\frac{\partial \mathbf{u}}{\partial E_{i}}\left(E=\mu_{E}\right), & \mathbf{u}_{, i j}=\frac{\partial^{2} \mathbf{u}}{\partial E_{i} \partial E_{j}}\left(E=\mu_{E}\right)
\end{array}
$$

Note that the Taylor expansion of $\mathbf{F}$ does not appear here, since the expression of $\mathbf{F}$ does not contain $\mathrm{E}$.

Substituting Eqs. (16a) and (16b) into Eq. (15) and collecting the terms of the same order yield

$$
\begin{aligned}
& \mathbf{K}_{0} \mathbf{u}_{0}=\mathbf{F} \\
& \mathbf{K}_{0} \mathbf{u}_{, i}=-\mathbf{K}_{, i} \mathbf{u}_{0} \\
& \mathbf{K}_{0} \mathbf{u}_{, i j}=-\mathbf{K}_{, i j} \mathbf{u}_{0}-\mathbf{K}_{, i} \mathbf{u}_{, i}-\mathbf{K}_{, j} \mathbf{u}_{, j} \text { or } \\
& \vdots \quad \vdots \\
& \mathbf{u}_{0}=\mathbf{K}_{0}^{-1} \mathbf{F} \\
& \mathbf{u}_{, i}=\mathbf{K}_{0}^{-1}\left(-\mathbf{K}_{, i} \mathbf{u}_{0}\right) \\
& \mathbf{u}_{, i j}=\mathbf{K}_{0}^{-1}\left(-\mathbf{K}_{, i j} \mathbf{u}_{0}-\mathbf{K}_{, i} \mathbf{u}_{, i}-\mathbf{K}_{, j} \mathbf{u}_{, j}\right) \\
& \vdots \quad \vdots \quad \vdots
\end{aligned}
$$

Eq. (18) is the SSMLPG formulation of Eq. (1). Applying this equation follows four steps: (1) Compute $\mathbf{F}$ and $\mathbf{K}_{0}$ by setting $\mathrm{E}_{\mathrm{J}}=\mu_{\mathrm{E}}\left(\mathrm{J}=1\right.$ to $\left.\mathrm{N}_{\mathrm{T}}\right)$; (2) Compute $\mathbf{u}_{0}=\mathbf{K}_{0}^{-1} \mathbf{F}$; (3) Compute $\mathbf{K}_{, \mathrm{i}}, \mathbf{K}_{\mathrm{ij}}, \ldots .$. using $\mathrm{E}_{\mathrm{J}}=\mu_{\mathrm{E}}(\mathrm{J}=1$ to $\mathrm{N}_{\mathrm{T}}$ ); and (4) Solve $\mathbf{u}_{, i}, \mathbf{u}_{, \mathrm{ij}} \ldots$ by the resulting $\mathbf{u}_{0}$. In addition, with setting $E_{J}=\mu_{E}\left(J=1\right.$ to $\left.N_{T}\right)$, any MLPG program can be used to complete the first three steps. An additional module can be appended to this MLPG package for solving $\mathbf{u}_{\mathrm{i}, \mathrm{i}}, \mathbf{u}_{, \mathrm{j}} \ldots$.

Furthermore, observing Eq. (18) can understand that the stability of Eq. (18) depends upon whether $\mathbf{K}_{, \mathrm{i}}, \mathbf{K}_{\text {,ij }}, \ldots$ can be obtained. For the current study, obtaining $\mathbf{K}_{, \mathrm{i}}, \mathbf{K}_{, \mathrm{ij}}, \ldots$ is easy, since only a linear elastic stress-strain relationship is introduced.

After accumulating the resulting $\mathbf{u}_{0}, \mathbf{u}_{, \mathrm{i}}, \mathbf{u}_{\mathrm{ij}} \ldots$, a first-order perturbation approximation of $\mathbf{u}$ is

$\mathbf{u}=\mathbf{u}_{0}+\sum_{i=1}^{N_{T}} \mathbf{u}_{, i} \Delta E_{i}$

with the expected value: $\operatorname{Ex}[\mathbf{u}]=\mathbf{u}_{0}$

and the covariance matrix:

$\operatorname{Cov}[\mathbf{u}, \mathbf{u}]=\operatorname{Ex}\left[(\mathbf{u}-\operatorname{Ex}[\mathbf{u}])(\mathbf{u}-\operatorname{Ex}[\mathbf{u}])^{T}\right]=\sum_{i=1}^{N_{T}} \sum_{j=1}^{N_{T}} \mathbf{u}_{, i}\left(\mathbf{u}_{, j}\right)^{T} \operatorname{Ex}\left[\Delta E_{i} \Delta E_{j}\right]$

in which $\operatorname{Ex}\left[\Delta \mathrm{E}_{\mathrm{i}} \Delta \mathrm{E}_{\mathrm{j}}\right]$ can be determined analytically by the autocorrelation function of E. In addition, a second-order perturbation approximation of $\mathbf{u}$ is
$\mathbf{u}=\mathbf{u}_{0}+\sum_{i=1}^{N_{T}} \mathbf{u}_{, i} \Delta E_{i}+\frac{1}{2} \sum_{i=1}^{N_{T}} \sum_{j=1}^{N_{T}} \mathbf{u}_{i j} \Delta E_{i} \Delta E_{j}$

with the expected value:

$\mathrm{E} x[\mathbf{u}]=\mathbf{u}_{0}+\frac{1}{2} \sum_{i=1}^{N_{T}} \sum_{j=1}^{N_{T}} \mathbf{u}_{, i j} \operatorname{Ex}\left[\Delta E_{i} \Delta E_{j}\right]$

and the covariance matrix:

$$
\begin{aligned}
& \operatorname{Cov}[\mathbf{u}, \mathbf{u}]=\sum_{i=1}^{N_{T}} \sum_{j=1}^{N_{T}} \mathbf{u}_{, i}\left(\mathbf{u}_{, j}\right)^{T} \operatorname{Ex}\left[\Delta E_{i} \Delta E_{j}\right] \\
& +\frac{1}{4} \sum_{i=1}^{N_{T}} \sum_{j=1}^{N_{T}} \sum_{k=1}^{N_{T}} \sum_{l=1}^{N_{T}} \mathbf{u}_{i j}\left(\mathbf{u}_{i j}\right)^{T}\left\{\begin{array}{l}
\mathrm{E} x\left[\Delta E_{i} \Delta E_{l}\right] \operatorname{Ex}\left[\Delta E_{j} \Delta E_{k}\right] \\
+\operatorname{Ex}\left[\Delta E_{i} \Delta E_{k}\right] \operatorname{Ex}\left[\Delta E_{j} \Delta E_{l}\right]
\end{array}\right\}
\end{aligned}
$$

In addition, observing Eqs. (19) and (22) can understand that the posterior errors of perturbation method are caused by truncating $\mathbf{u}_{\mathrm{ijk}}, \mathbf{u}_{\mathrm{ijkL}} \ldots\left(\mathrm{i}, \mathrm{j}, \mathrm{k}, \mathrm{L}=1\right.$ to $\mathrm{N}_{\mathrm{T}}$ ) in equating these two equations. Additionally computing these $\mathbf{u}_{, \mathrm{ijk}}, \mathbf{u}_{, \mathrm{ijkL}} \ldots$ can estimate those posterior errors.

\section{RESULTS}

Implement the performance evaluation of proposed perturbation-based SSMLPG formulation through a stochastic elastostatic problem in which a strip loading bears on a field foundation clay layer. As a comparison, an SSFEM package FERUM [9] is additionally applied to this stochastic elastostatic problem. Eqs. (19), (22) and the FERUM package are used to predict $\operatorname{Ex}[\mathbf{u}]$ and standard deviation $\operatorname{Std}[\mathbf{u}]$ with the uncertainty in the spatial variability of E. Note that the FERUM package does not include any module for compute the perturbation expansion of a random field. Therefore, an additional module is appended to the FERUM package for providing the perturbation-based SSFEM results.

For simplicity, the performance evaluation of perturbation-based SSMLPG formulation focuses on three aspects:

a. Comparing the agreements between the MCS and SSMLPG or SSFEM results.

b. Evaluating the influence of the spatial variability of $\mathrm{E}$ on the agreements between the MCS and SSMLPG results.

c. Studying the agreements between the MCS and SSMLPG results when discrete nodes are used.

Fig. (2) displays the layout of $\Omega$ and boundary conditions in which B is the half of foundation width, $\mathrm{H}$ is the thickness of the clay layer, and $\sigma_{0}$ is the magnitude of the foundation loading. In addition, assume the spatial variability of $E$ follows two probabilistic distributions (named by Distributions A and B). The first probabilistic distribution is

$E=\mu_{E}[1+\alpha(\mathbf{x})]$

where $\mu_{\mathrm{E}}$ is independent of $\mathbf{x}$ and $\alpha(\mathbf{x})$ is a zero-mean, scalar, homogeneous random field with its autocorrelation function $\Gamma_{\alpha}(\xi)$ equal to 


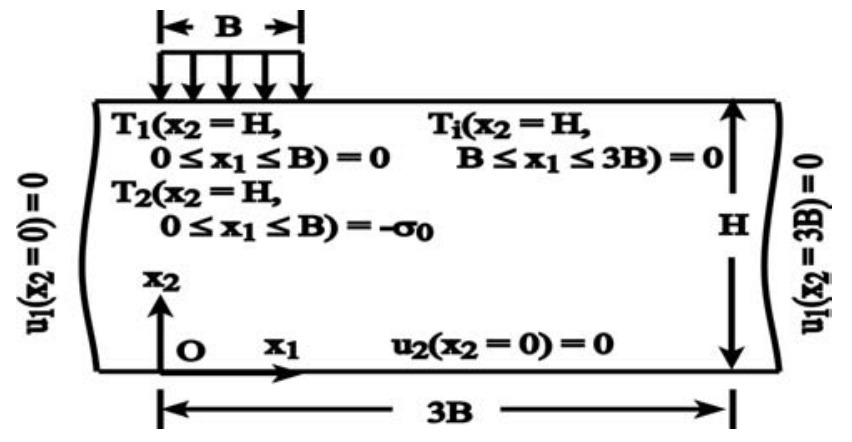

Fig. (2).Benchmark problem for implementing a performance evaluation of the perturbation-based SSMLPG formulation (not to scale).

(Distribution A) $\Gamma_{\alpha}(\xi)=S_{E}^{2} \exp \left[-\left(\frac{\left|\xi_{1}\right|}{b_{1} B}+\frac{\left|\xi_{2}\right|}{b_{2} H}\right)\right]$

where $b_{1}$ and $b_{2}$ are two constants, $S_{E}$ is the standard deviation of $\mathrm{E},\left(\xi_{1}, \xi_{2}\right)=\mathbf{x}_{2}-\mathbf{x}_{1}$, and $\mathbf{x}_{\mathrm{i}}, \mathrm{i}=1$ to 2 are two nodes. Based on Eq. (26), $\operatorname{Ex}\left[\Delta \mathrm{E}_{\mathrm{i}} \Delta \mathrm{E}_{\mathrm{j}}\right]$ is derived by

$$
\operatorname{Ex}\left[\Delta E_{i} \Delta E_{j}\right]=S_{E}^{2} \exp \left[-\left(\frac{\left|x_{i, 1}-x_{j, 1}\right|}{b_{1} B}+\frac{\left|x_{i, 2}-x_{j, 2}\right|}{b_{2} H}\right)\right]
$$

where $\mathrm{x}_{\mathrm{i}, 1}$ and $\mathrm{x}_{\mathrm{i}, 2}$ are the components of $\mathbf{x}_{\mathrm{i}}, \mathrm{x}_{\mathrm{j}, 1}$ and $\mathrm{x}_{\mathrm{j}, 2}$ are the components of $\mathbf{x}_{\mathbf{j}}$. Similarly manipulating Eq. (27) can obtain $\operatorname{Ex}\left[\Delta \mathrm{E}_{\mathrm{i}} \Delta \mathrm{E}_{1}\right], \quad \operatorname{Ex}\left[\Delta \mathrm{E}_{\mathrm{j}} \Delta \mathrm{E}_{\mathrm{k}}\right], \quad \operatorname{Ex}\left[\Delta \mathrm{E}_{\mathrm{i}} \Delta \mathrm{E}_{\mathrm{k}}\right], \quad$ and $\operatorname{Ex}\left[\Delta \mathrm{E}_{\mathrm{j}} \Delta \mathrm{E}_{1}\right]$.

The second probability distribution is similar to Eq. (25) except that $\Gamma_{\alpha}(\xi)$ is

(Distribution B) $\Gamma_{\alpha}(\xi)=S_{E}^{2}\left(1-\frac{\left|\xi_{1}\right|}{b_{1} B}\right)\left(1-\frac{\left|\xi_{2}\right|}{b_{2} H}\right)$

Based on Eq. (28), $\operatorname{Ex}\left[\Delta \mathrm{E}_{\mathrm{i}} \Delta \mathrm{E}_{\mathrm{j}}\right]$ is re-derived by

$$
\operatorname{Ex}\left[\Delta E_{i} \Delta E_{j}\right]=S_{E}^{2}\left(1-\frac{\left|x_{i, 1}-x_{j, 1}\right|}{b_{1} B}\right)\left(1-\frac{\left|x_{i, 2}-x_{j, 2}\right|}{b_{2} H}\right)
$$

However, since $\mathrm{E}$ varies according to a random field $\alpha(\mathbf{x})$, the analytical solutions of $\mathbf{u}$ don't exist. We should generate an MCS to provide the standard for comparing the SSMLPG and SSFEM results. Implementing an MCS requires three steps: (a) Generate a number of samples of $\mathrm{E}$ according to Eq. (26) or (28); and (b) Substitute each sample of E into Eq. (15) and the FERUM package to predict $\mathbf{u}$. (c) Compute $\operatorname{Ex}[\mathbf{u}]$ and $\operatorname{Std}[\mathbf{u}]$ values of the resulting $\mathbf{u}$. If the total number of samples of $\mathrm{E}$ is sufficiently large, the resulting Ex[u] and Std[u] values approach their exact values (but are still not exact values). Such $\operatorname{Ex}[\mathbf{u}]$ and $\operatorname{Std}[\mathbf{u}]$ values can be the standard for comparing the SSFEM-based and SSMLPG-based predicted $\operatorname{Ex}[\mathbf{u}]$ and $\operatorname{Std}[\mathbf{u}]$. Essential parameters for this comparison are listed below

a. Set $\sigma_{0}=1 \mathrm{kN} / \mathrm{m}^{2}, v=0.3, \mathrm{~B}=10 \mathrm{~m}$, and $\mathrm{H}=4.3 \mathrm{~m}$. Define $\Omega$ as $0 \leq \mathrm{x}_{1} \leq 3 \mathrm{~B}$ and $0 \leq \mathrm{x}_{2} \leq \mathrm{H}$. Generate 15000 samples of $E$ to implement an MCS. Adopt 16 quadrature points in calculating the numerical integration over an $\Omega_{\mathrm{S}}$.

b. Set $\alpha_{c}=4.0, q=1.03$, and $d_{c}=3.0$ in computing the MQ RBF. These $\alpha_{c}, q$, and $d_{c}$ values come from a published reference [10] in which the most proper $\alpha_{c}$, $\mathrm{q}$, and $\mathrm{d}_{\mathrm{c}}$ values for applying this MQ RBF are studied. As a consequence, setting $\alpha_{c}=4.0, q=1.03$, and $\mathrm{d}_{\mathrm{c}}=3.0$ results in satisfactory interpolation errors.

c. Intentionally choose a complete monomial basis of order 3 to construct $\phi$. Observe the agreement between the corresponding MCS and SSMLPG results with respect to such a lower order value.

d. Choose $\Omega_{\mathrm{Q}}$ for any point as a circle centered at this point. Set the radius of each $\Omega_{\mathrm{Q}}$ equal to $2.58 \mathrm{~m}$. Choose $\Omega_{\mathrm{S}}$ for a node as a rectangle centered at this node. Set the width and length of each $\Omega_{\mathrm{S}}$ both equal to $2.58 \mathrm{~m}$. Such sizes of $\Omega_{\mathrm{Q}}$ and $\Omega_{\mathrm{S}}$ are set according to a previous study [10] and a pilot test evaluating the time spent to complete an MCS. Accordingly, the size of $\Omega_{\mathrm{Q}}$ or $\Omega_{\mathrm{S}}$ should be larger than 1.5 times the spacing of any two connecting nodes for obtaining less interpolation errors [10]. Meanwhile, the pilot test identified the time spent to complete an MCS using 15000 samples is acceptable ${ }^{1}$.

e. Generate two cases (named by Discretizations A and B) of meshless discretization. Fig. (3a, b) illustrate these two cases of the meshless discretization. Fig. (3c) shows the finite element discretization for executing the FERUM package.

f. Define the accuracy standard is $\varepsilon\left\{\operatorname{Ex}\left[\mathrm{u}_{\mathrm{i}}\right]\right\} \leq 10 \%$, $\varepsilon\left\{\operatorname{Std}\left[\mathrm{u}_{\mathrm{i}}\right]\right\} \leq 10 \%, \Delta\left\{\operatorname{Ex}\left[\mathrm{u}_{\mathrm{i}}\right]\right\} \leq 10 \%$, and $\Delta\left\{\operatorname{Std}\left[\mathrm{u}_{\mathrm{i}}\right]\right\}$ $\leq 10 \%$ where $\operatorname{Ex}\left[\mathrm{u}_{\mathrm{i}}\right]$ and $\operatorname{Std}\left[\mathrm{u}_{\mathrm{i}}\right]$ are; respectively, the components of $\operatorname{Ex}[\mathbf{u}]$ and $\operatorname{Std}[\mathbf{u}], \varepsilon$ and $\Delta$ are the error estimators quantifying the agreements between MCS and SSMLPG or SSFEM results. $\varepsilon$ and $\Delta$ are defined by

$$
\begin{aligned}
& \varepsilon\left\{\operatorname{Ex}\left[u_{i}\right]\right\}(\%)=\left|\frac{E x\left[u_{i}\right]_{M C S}-E x\left[u_{i}\right]_{S S M P G}}{E x\left[u_{i}\right]_{M C S}}\right|, \varepsilon\left(\operatorname{Std}\left[u_{i}\right]\right)(\%)=\left|\frac{\operatorname{Std}\left[u_{i}\right]_{M C S}-\operatorname{St}\left[u_{i}\right]_{S S M L G G}}{\operatorname{Std}\left[u_{i}\right]_{M C S}}\right| \\
& \Delta\left\{E x\left[u_{i}\right]\right\}(\%)=\left|\frac{E x\left[u_{i}\right]_{\text {MCS }}-E x\left[u_{i}\right]_{S S E M}}{E x\left[u_{i}\right]_{\text {MSS }}}\right|, \Delta\left\{\operatorname{Std}\left[u_{i}\right]\right\}(\%)=\left|\frac{\operatorname{Std}\left[u_{i}\right]_{\text {MCS }}-\operatorname{Std}\left[u_{i}\right]_{S S E M U}}{\operatorname{Std}\left[u_{i}\right]_{\text {MCS }}}\right|
\end{aligned}
$$

In Eqs. (30a) and (30b) $\operatorname{Ex}\left[\mathrm{u}_{\mathrm{i}}\right]_{\mathrm{MCS}}$ and $\operatorname{Std}\left[\mathrm{u}_{\mathrm{i}}\right]_{\mathrm{MCS}}$ are computed by

$$
\operatorname{Ex}\left[u_{i}\right]=\frac{1}{N_{s}} \sum_{j=1}^{N_{s}} u_{i, j}, \operatorname{Std}\left[u_{i}\right]=\sqrt{\frac{1}{N_{s}} \sum_{j=1}^{N_{s}}\left\{u_{i, j}-\operatorname{Ex}\left[u_{i}\right]\right\}^{2}}
$$

\footnotetext{
${ }^{1}$ Using an Intel ${ }^{\circledR}$ Core $^{\mathrm{TM}} 2$ Duo CPU.
} 

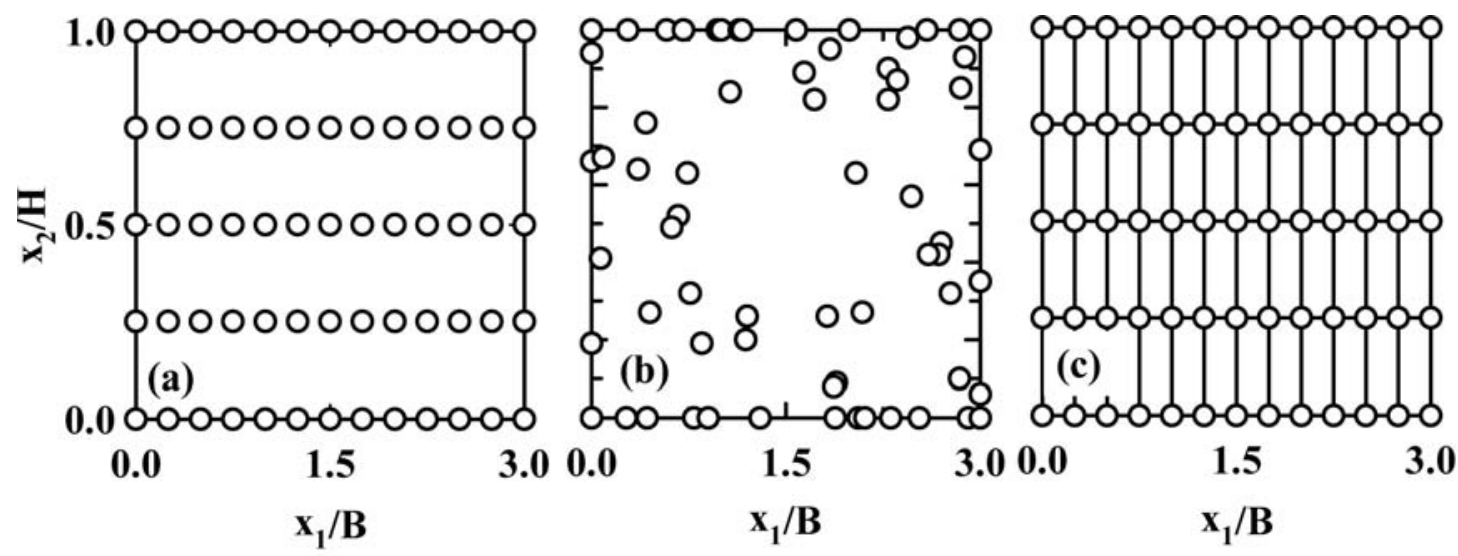

Discretization A: meshless discretization of 65 equally spaced nodes

\section{Discretization B: meshless finite element discretization discretization of 65 discete nodes \\ (65 uniformly distributed nodes and 48 linear quadrilateral elements)}

Fig. (3).Meshless and finite element discretizations for analyzing the benchmark problem (not to scale).

where, $\mathrm{N}_{\mathrm{s}}$ is the total number of samples of $\mathbf{u}$, and the subscript $\mathrm{j}$ denotes the $\mathrm{j}$-th sample of $\mathrm{E}$. In addition, Ex $\left[\mathrm{u}_{\mathrm{i}}\right]_{\text {SSMLPG }}$ and Ex $\left[\mathrm{u}_{\mathrm{i}}\right]_{\text {SSFEM }}$ can be calculated by Eq. (20) or (23). However, $\operatorname{Std}\left[u_{i}\right]_{\text {SSMLPG }}$ and $\operatorname{Std}\left[u_{i}\right]_{\text {SSFEM }}$ are computed by

$\operatorname{Var}(\mathbf{u})=\operatorname{diag}\{\operatorname{Cov}[\mathbf{u}, \mathbf{u}]\}, \operatorname{Std}\left[u_{i}\right]=\sqrt{\operatorname{Var}\left(u_{i}\right)}$

where $\operatorname{Var}(\mathbf{u})$ is the variance of $\mathbf{u}, \operatorname{Var}\left(\mathrm{u}_{\mathrm{i}}\right)$ are its components, and diag is the diagonals of $\operatorname{Cov}[\mathbf{u}, \mathbf{u}]$.
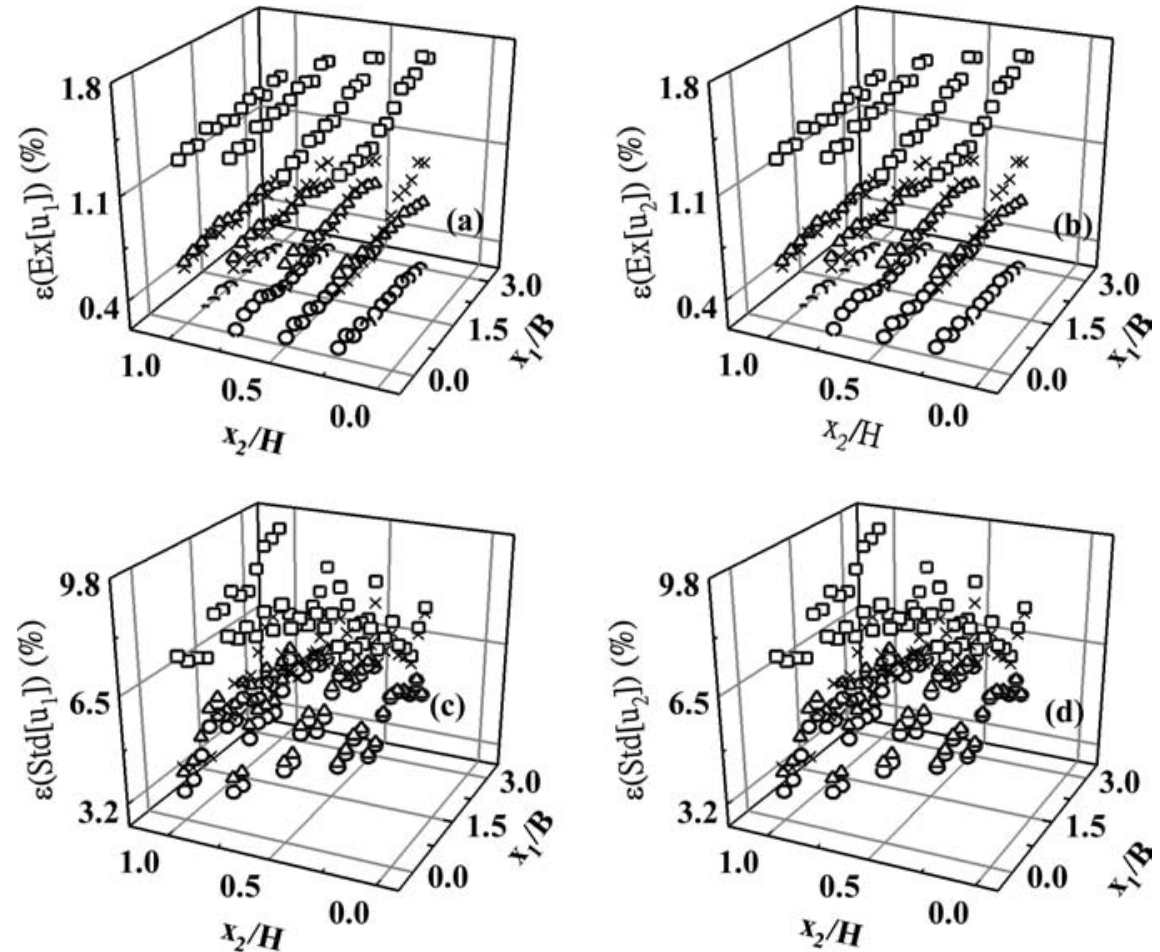

$$
\begin{array}{ll}
\hline \text { Eq.(19) (Distribution A) } & \Delta \text { Eq.(19) (Distribution B) } \\
\times \text { Eq.(22) (Distribution A) } & \text { O Eq.(22) (Distribution B) }
\end{array}
$$

Fig. (4).Variation of $\varepsilon\left(\operatorname{Ex}\left[u_{i}\right]\right)$ and $\varepsilon\left(\operatorname{Std}\left[u_{i}\right]\right)(i=1$ to 2$)$ versus $x_{i}\left(\right.$ Discretization $\left.A, S_{E} / \mu_{E}=0.12, b_{1}=b_{2}=1, N_{s}=15000\right)$. 

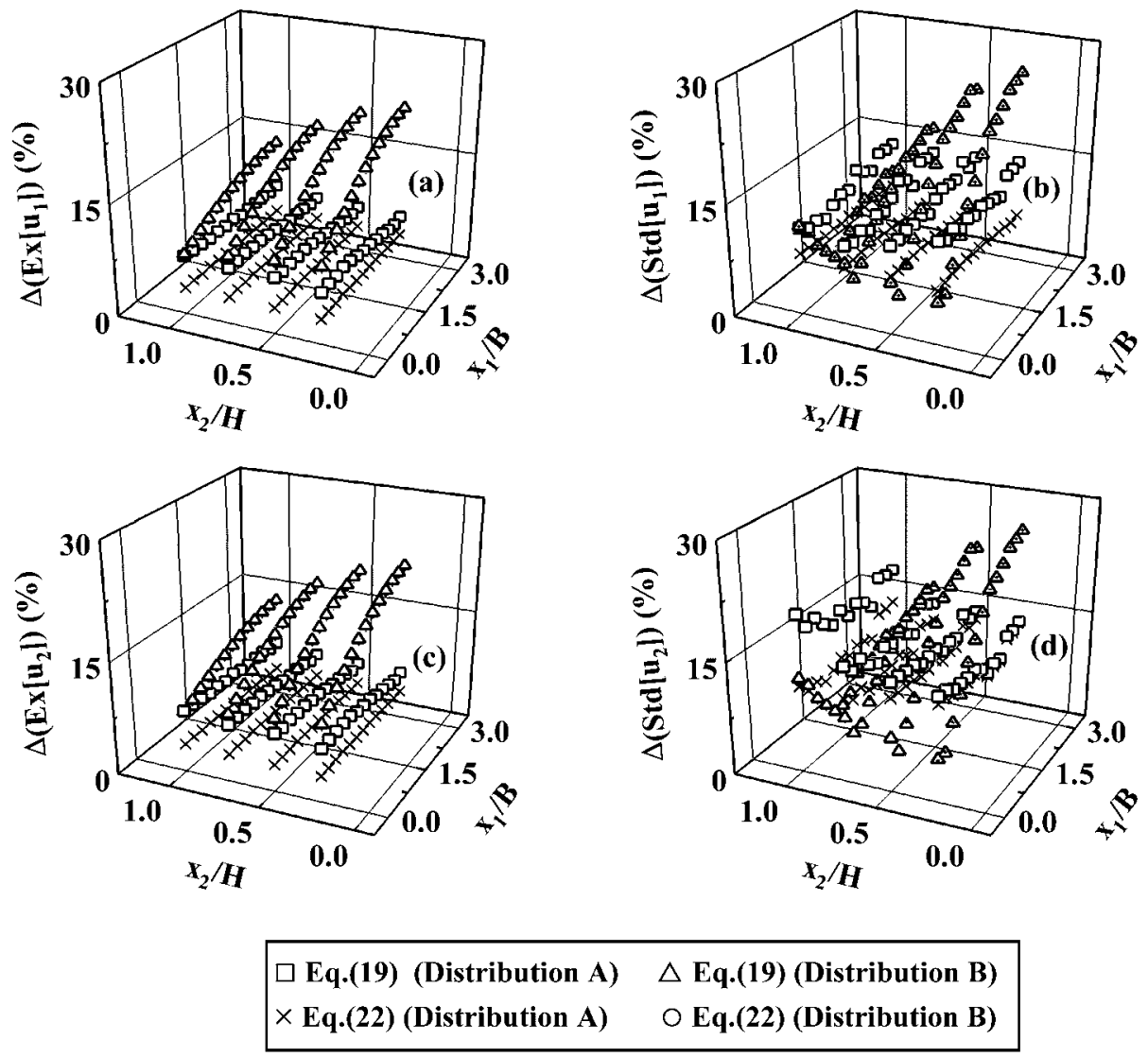

Fig. (5).Variation of $\Delta\left(\operatorname{Ex}\left[\mathrm{u}_{\mathrm{i}}\right]\right)$ and $\Delta\left(\operatorname{Std}\left[\mathrm{u}_{\mathrm{i}}\right]\right)(\mathrm{i}=1$ to 2$)$ versus $\mathrm{x}_{\mathrm{i}}\left(\mathrm{S}_{\mathrm{E}} / \mu_{\mathrm{E}}=0.12, \mathrm{~b}_{1}=\mathrm{b}_{2}=1, \mathrm{~N}_{\mathrm{s}}=15000\right)$.

Table 1. Ranges of $\varepsilon\left(\operatorname{Ex}\left[u_{i}\right]\right), \varepsilon\left(\operatorname{Std}\left[u_{i}\right]\right), \Delta\left(\operatorname{Ex}\left[u_{i}\right]\right)$ and $\Delta\left(\operatorname{Std}\left[u_{i}\right]\right)(i=1$ to 2$)$

\begin{tabular}{|l|l|c|c|c|c|c|c|c|c|}
\hline \multicolumn{2}{|c|}{} & $\boldsymbol{\varepsilon}\left(\mathbf{E x}\left[\mathbf{u}_{1}\right]\right)$ & $\boldsymbol{\varepsilon}\left(\mathbf{E x}\left[\mathbf{u}_{2}\right]\right)$ & $\boldsymbol{\varepsilon}\left(\mathbf{S t d}\left[\mathbf{u}_{1}\right]\right)$ & $\boldsymbol{\varepsilon}\left(\mathbf{S t d}\left[\mathbf{u}_{2}\right]\right)$ & $\Delta\left(\operatorname{Ex}\left[\mathbf{u}_{1}\right]\right)$ & $\Delta\left(\operatorname{Ex}\left[\mathbf{u}_{2}\right]\right)$ & $\Delta\left(\operatorname{Std}\left[\mathbf{u}_{1}\right]\right)$ & $\Delta\left(\mathbf{S t d}\left[\mathbf{u}_{2}\right]\right)$ \\
\hline \hline \multirow{2}{*}{ 1st-order (\%) } & Distribution A & $1.31 \sim 1.72$ & $1.3 \sim 1.71$ & $6.49 \sim 9.43$ & $6.49 \sim 9.44$ & $5.97 \sim 7.92$ & $6.23 \sim 8.28$ & $12.1 \sim 22.1$ & $10.7 \sim 20.8$ \\
\cline { 2 - 10 } & Distribution B & $0.63 \sim 0.82$ & $0.62 \sim 0.81$ & $4.03 \sim 6.73$ & $4.02 \sim 6.73$ & $8.13 \sim 21.4$ & $8.13 \sim 21.3$ & $4.1 \sim 26$ & $4.44 \sim 26.1$ \\
\hline \multirow{2}{*}{ 2nd-order (\%) } & Distribution A & $0.59 \sim 1.01$ & $0.6 \sim 1.01$ & $3.84 \sim 8.08$ & $3.85 \sim 8.09$ & $3.38 \sim 3.86$ & $3.48 \sim 3.95$ & $9.89 \sim 14.6$ & $9.41 \sim 13.7$ \\
\cline { 2 - 9 } & Distribution B & $0.13 \sim 0.32$ & $0.12 \sim 0.31$ & $3.42 \sim 6.41$ & $3.42 \sim 6.41$ & $7.65 \sim 21$ & $7.64 \sim 20.9$ & $4.03 \sim 21$ & $4.03 \sim 22.1$ \\
\hline
\end{tabular}

Surprisingly, Fig. (4a-d) identify that $\varepsilon\left(\operatorname{Ex}\left[\mathrm{u}_{\mathrm{i}}\right]\right)$ and $\varepsilon\left(\operatorname{Std}\left[u_{i}\right]\right)$ computed based on Eq. (19) satisfy the accuracy standard. In addition, comparing Table 1, Figs. (4a-d, 5a-d) indicates that the SSMLPG results approach more closely to the MCS results than the SSFEM results do, since $\varepsilon\left(\operatorname{Ex}\left[\mathrm{u}_{\mathrm{i}}\right]\right)$ and $\varepsilon\left(\operatorname{Std}\left[u_{i}\right]\right)$ are; respectively, less than $\Delta\left(\operatorname{Ex}\left[u_{i}\right]\right)$ and $\Delta\left(\operatorname{Std}\left[\mathrm{u}_{\mathrm{i}}\right]\right)$. Note that generating the SSMLPG results spends about 300 seconds; while completing the MCS requires about $1.5 \mathrm{hr}$.

\section{Influence of the Spatial Variability of Young's Modulus on the Spectral Stochastic Meshless Local Petrov- Galerkin Results}

Discuss first the necessity of accounting for the spatial variability of $E$ in predicting $\mathbf{u}$. As an example, Fig. (6a, b) display variation of $\mathrm{u}_{\mathrm{i}}\left(\mathrm{x}_{2}=\mathrm{H} \wedge 0 \leq \mathrm{x}_{1} \leq 3 \mathrm{~B}\right) / \mathrm{H}$ (i=1 to 2$) v s$ $\mathrm{x}_{1}$ with and without accounting for the spatial variability of E. In these two figures, $\mathbf{u}$ is predicted by Eq. (19).

As compared to SSMLPG-based predicted $\mathrm{u}_{1}\left(\mathrm{x}_{2}=\mathrm{H}\right) / \mathrm{H}$, Fig. $(\mathbf{6 a}, \mathbf{b})$ demonstrate that SSMLPG-based predicted $\mathrm{u}_{2}\left(\mathrm{x}_{2}\right.$ $=\mathrm{H}) / \mathrm{H}$ varies within a wider range. As a consequence, obtaining unreliable predicted $\mathrm{u}_{2}\left(\mathrm{x}_{2}=\mathrm{H}\right)$ is more possible than obtaining unreliable predicted $u_{1}\left(x_{2}=H\right)$, if the spatial variability of $\mathrm{E}$ is neglected.

Next, study the influence of different spatial variability of E on the accuracy of SSMLPG results. Since Eqs. (26) and (28) show that adjusting the spatial variability of $\mathrm{E}$ is equivalent to using different $b_{1}, b_{2}$, and $S_{E}$ values, we turn to study the influence of different $b_{1}, b_{2}$, and $S_{E}$ values on the agreements between the MCS and SSMLPG results. Fig. (7a, b) show variation of $\varepsilon\left(\operatorname{Ex}\left[\mathrm{u}_{\mathrm{i}}\right]\right)$ and $\varepsilon\left(\operatorname{Std}\left[\mathrm{u}_{\mathrm{i}}\right]\right)(\mathrm{i}=1$ to 2$)$ vs $\mathrm{x}_{\mathrm{i}}, \mathrm{b}_{1}=1$, and $\mathrm{b}_{2}=2$. Fig. $(\mathbf{8 a}, \mathbf{b})$ show variation of 

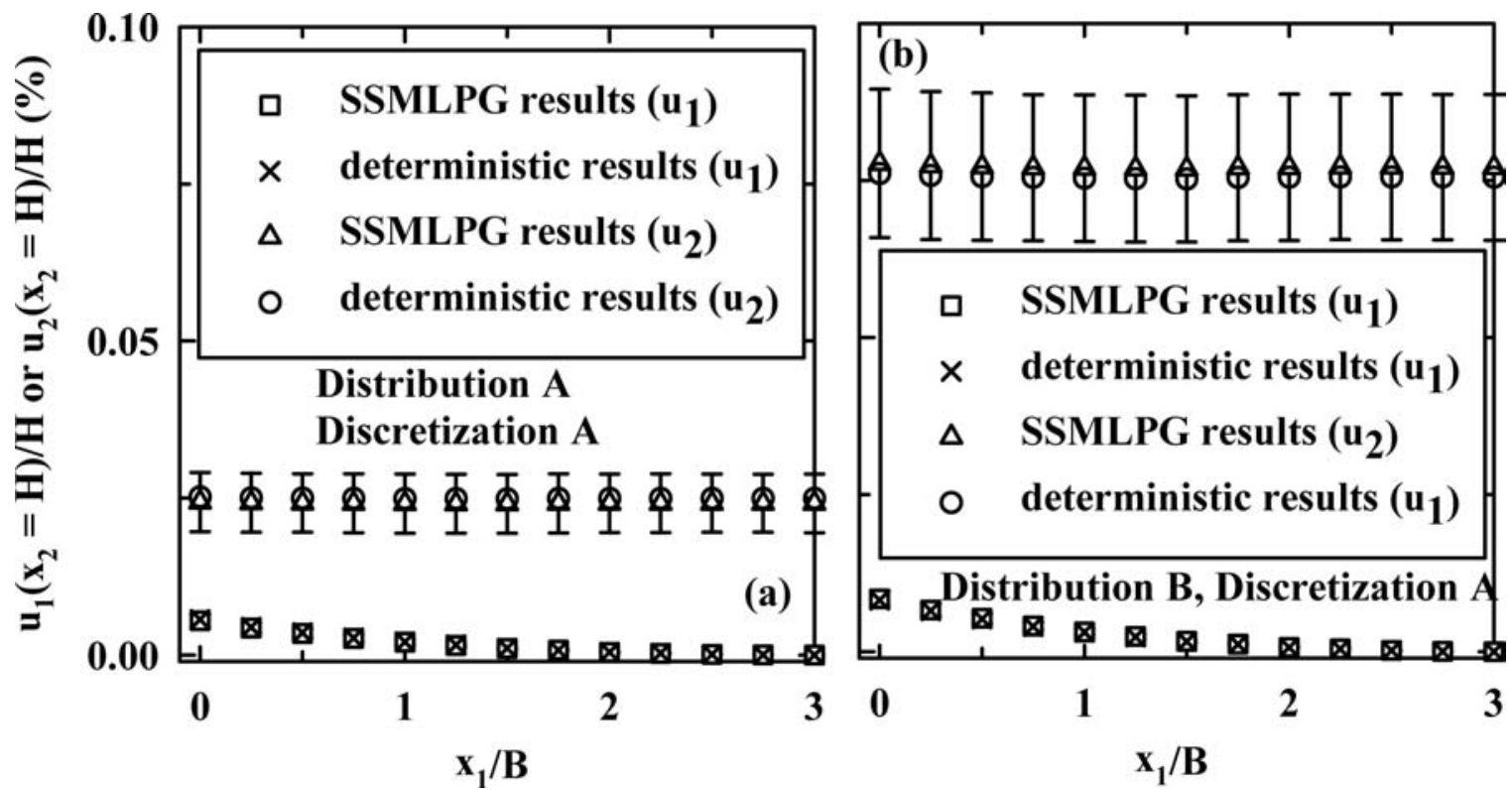

Fig. (6).Comparison of predicted $\mathbf{u}\left(\mathrm{x}_{2}=\mathrm{H} \wedge 0 \leq \mathrm{x}_{1} \leq 3 \mathrm{~B}\right) / \mathrm{H}$ with and without accounting for the uncertainty contributed by the spatial variability of E (using Eq. (19)).

$\varepsilon\left(\operatorname{Ex}\left[\mathrm{u}_{\mathrm{i}}\right]\right)$ and $\varepsilon\left(\operatorname{Std}\left[\mathrm{u}_{\mathrm{i}}\right]\right) v s \mathrm{x}_{\mathrm{i}}$ and $\mathrm{S}_{\mathrm{E}} / \mu_{\mathrm{E}}=0.24$. In these four figures, $\mathbf{u}$ is computed by Eq. (22).

Comparing Figs. (4c, d, 7a, b) identifies that $\varepsilon\left(\operatorname{Ex}\left[\mathrm{u}_{\mathrm{i}}\right]\right)$ and $\varepsilon\left(\operatorname{Std}\left[u_{i}\right]\right)$ values decrease when $b_{2}$ values increase. Comparing Figs. (4c, $\mathbf{d}, \mathbf{8 a}, \mathbf{b})$ indicates that doubling the $\mathrm{S}_{\mathrm{E}}$ value increases the $\varepsilon\left(\operatorname{Ex}\left[\mathrm{u}_{\mathrm{i}}\right]\right)$ and $\varepsilon\left(\operatorname{Std}\left[\mathrm{u}_{\mathrm{i}}\right]\right)$ values. In Fig. $(\mathbf{8 a}, \mathbf{b}), \varepsilon\left(\operatorname{Ex}\left[\mathrm{u}_{\mathrm{i}}\right]\right)$ values still satisfy the accuracy standard, whereas some of $\varepsilon\left(\operatorname{Std}\left[u_{i}\right]\right)$ values don't.

\section{Spectral Stochastic Meshless Local Petrov-Galerkin Results vs Discrete Nodes}

Using Eq. (22) and Discretization B, Fig. (9a, b) display variation of $\varepsilon\left(\operatorname{Ex}\left[\mathrm{u}_{\mathrm{i}}\right]\right)$ and $\varepsilon\left(\operatorname{Std}\left[\mathrm{u}_{\mathrm{i}}\right]\right)(\mathrm{i}=1$ to 2$) v s \mathrm{x}_{\mathrm{i}}$. These two figures indicate that the proposed perturbation-based

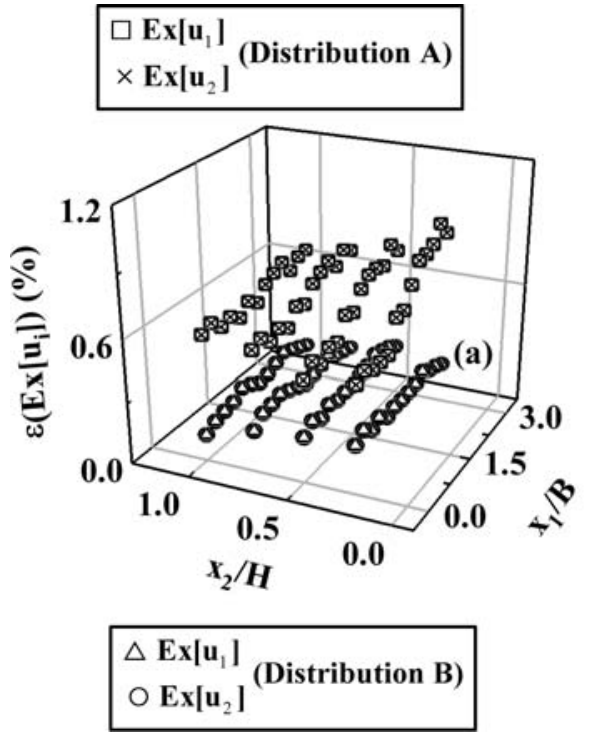

SSMLPG formulation can provide sufficiently accurate numerical results, even if nodes are randomly distributed. $\varepsilon\left(\operatorname{Ex}\left[u_{i}\right]\right)$ and $\varepsilon\left(\operatorname{Std}\left[u_{i}\right]\right)$ values in Fig. (9a, b) satisfy the accuracy standard.

\section{DISCUSSIONS AND CONCLUSION}

This study presents an SSMLPG formulation containing perturbation expansions of random fields and a 2D MWS formulation in elasticity. In Sec.4, the performance of this SSMLPG formulation is evaluated. Some discussions are drawn from the evaluation results:

a. Even if the first-order perturbation approximation of $\mathbf{u}$ is used, Fig. (4a-d) show that SSMLPG-based predicted $\operatorname{Ex}[\mathbf{u}]$ and $\operatorname{Std}[\mathbf{u}]$ are still sufficiently

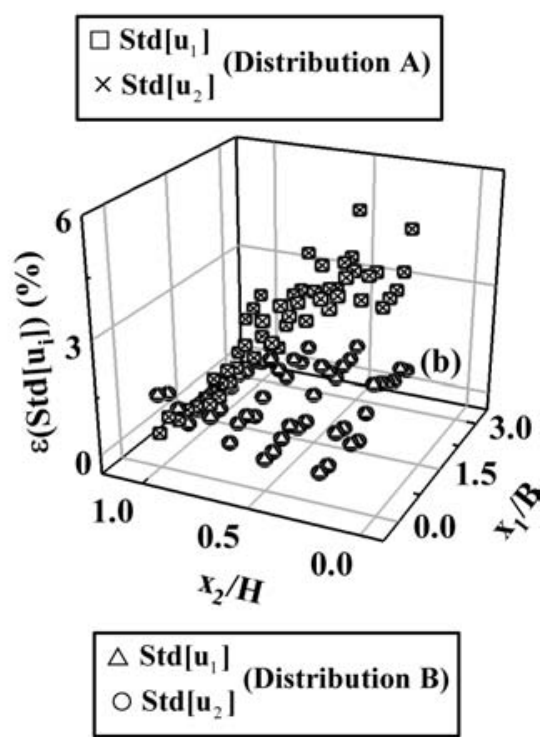

Fig. (7).Variation of $\varepsilon\left(\operatorname{Ex}\left[\mathrm{u}_{\mathrm{i}}\right]\right)$ and $\varepsilon\left(\operatorname{Std}\left[\mathrm{u}_{\mathrm{i}}\right]\right)(\mathrm{i}=1$ to 2$)$ versus $\mathrm{x}_{\mathrm{i}}$ and $\mathrm{b}_{1}=1, \mathrm{~b}_{2}=2$ (Using Eq. (22) Discretization $\mathrm{B}, \mathrm{S}_{\mathrm{E}} / \mu_{\mathrm{E}}=0.12$, and $\mathrm{N}_{\mathrm{s}}=$ 15000). 

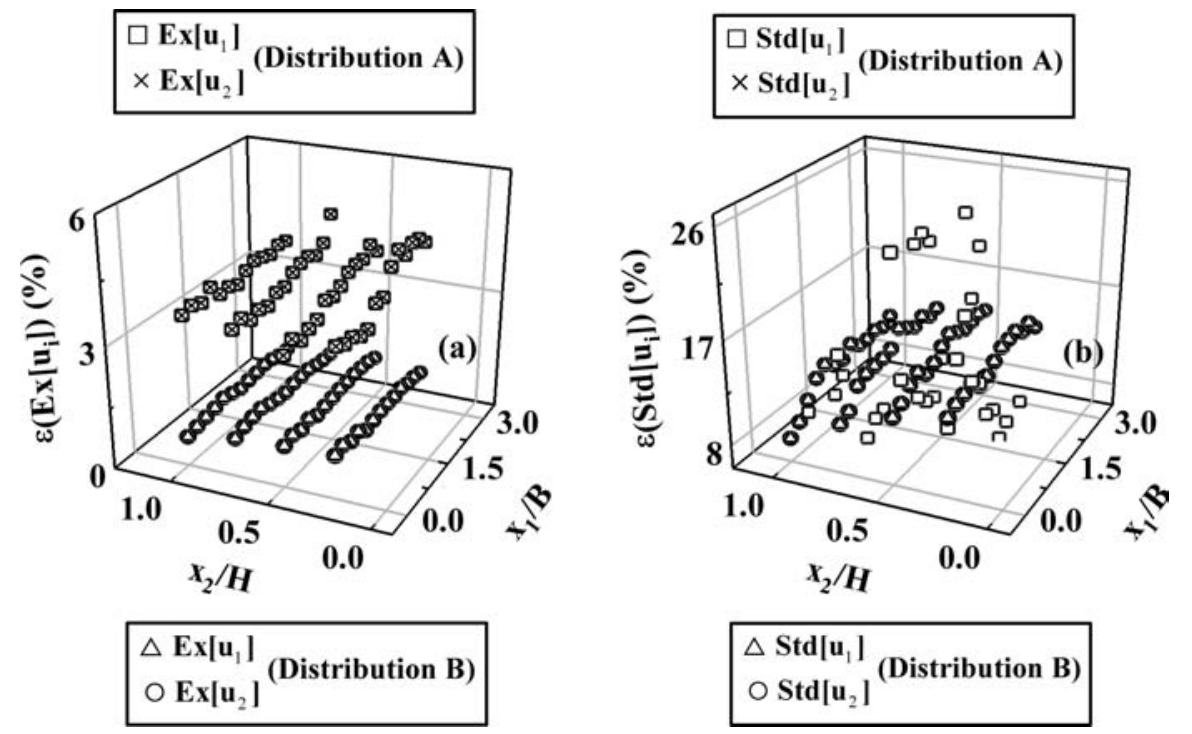

Fig. (8).Variation of $\varepsilon\left(\operatorname{Ex}\left[u_{i}\right]\right)$ and $\varepsilon\left(\operatorname{Std}\left[u_{i}\right]\right)(i=1$ to 2$)$ with respect to $x_{i}$ and $S_{E} / \mu_{E}=0.24$ (Using Eq. (22) Discretization $A$, $b_{1}=b_{2}=1$, and $\left.\mathrm{N}_{\mathrm{s}}=15000\right)$.
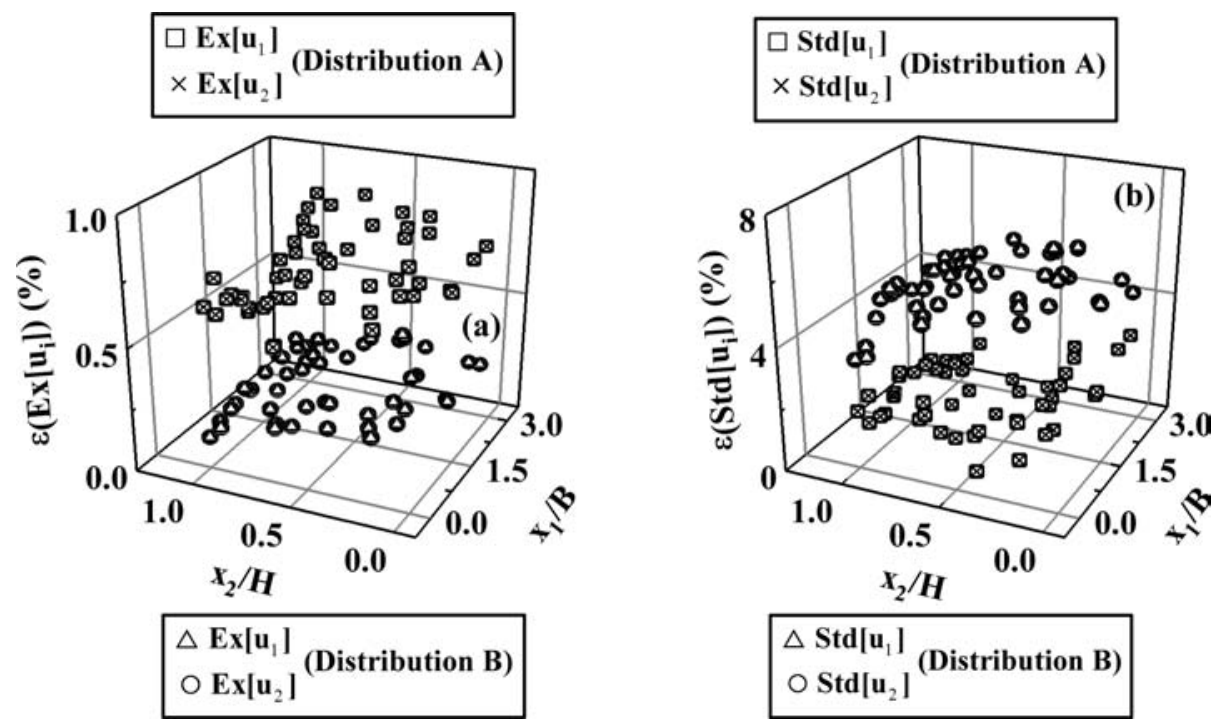

Fig. (9).Variation of $\varepsilon\left(\operatorname{Ex}\left[u_{i}\right]\right)$ and $\varepsilon\left(\operatorname{Std}\left[u_{i}\right]\right)(i=1$ to 2$)$ versus $x_{i}$ (Using Eq. (22) Discretization $B, S_{E} / \mu_{E}=0.12, b_{1}=b_{2}=1$, and $N_{s}=$ $15000)$.

accurate. Such experience was not mentioned in applying other stochastic numerical methods. For example, a previous study [11] concluded that the accuracy of stochastic element-free Galerkin (SEFGM)-based predicted $\operatorname{Ex}[\mathbf{u}]$ and $\operatorname{Std}[\mathbf{u}]$ is unsatisfactory, if the first-order perturbation approximation of $\mathbf{u}$ is used. Consequently, Fig. (4a-d) encourage the further application of perturbationbased SSMLPG formulation to three-dimensional problems. Solving a three-dimensional problem is usually time-consuming; whereas computing the firstorder perturbation approximation is time-saving. If using the first-order perturbation approximation of $\mathbf{u}$ can result in sufficiently accurate predicted $\operatorname{Ex}[\mathbf{u}]$ and $\operatorname{Std}[\mathbf{u}]$, we are more willing to apply the SSMLPG method to three-dimensional problems. b. Comparing Fig. (4a-d) with Fig. (5a-d) identifies that the SSMLPG results approach more closely to the MCS results than the SSFEM results do. The better accuracy of SSMLPG results may attribute to the difference between meshfree and finite element shape functions, since other parameters for plotting Figs. (4a-d, 5a-d) are almost identical. Nevertheless, further applying the SSMLPG and SSFEM methods to other types of stochastic boundary-value problems is suggested.

c. Figs. (7a, b, 8a, b) show that $b_{1}, b_{2}$, and $S_{E}$ values do affect the accuracy of SSMLPG results. To obtain more proper $b_{1}, b_{2}$, and $S_{E}$ values, we may compute different $b_{1}, b_{2}$, and $S_{E}$ values for each $\Omega_{Q}$. Such an idea can be tested in solving a practical stochastic boundary-value problem. The SSMLPG method gives 
more freedom to support this idea, since each $\Omega_{\mathrm{Q}}$ is arbitrary. We can freely adjust any $\Omega_{\mathrm{Q}}$ but fix other $\Omega_{\mathrm{Q}}$ for obtaining more proper $\mathrm{b}_{1}, \mathrm{~b}_{2}$, and $\mathrm{S}_{\mathrm{E}}$ values.

d. Fig. (9a, b) display that the proposed perturbationbased SSMLPG formulation still provides satisfactory numerical results when discrete nodes are used. Such performance facilitates solving practical stochastic boundary-value problems. In a practical stochastic boundary-value problem, data of material properties are measured at discrete nodes. The SSMLPG method can directly incorporate with such data to produce sufficiently accurate numerical results. If the SSFEM and SEFGM methods are applied for the same stochastic boundary-value problem, additional nodes are required to create a finite element discretization or background cells.

In conclusion, the SSMLPG method can be an efficient alternative for solving stochastic boundary-value problems.

\section{ACKNOWLEDGEMENT}

Declared none.

\section{CONFLICT OF INTEREST}

Declared none.

\section{REFERENCES}

[1] Ghanem RG, Spanos PD. Stochastic finite elements: a spectral approach. New York: Springer 1981.

[2] Wu SQ, Law SS. Dynamic analysis of bridge with non-Gaussian uncertainties under a moving vehicle. Probab Eng Mech 2011; 26(2): 281-93.

[3] Ngah MF, Young A. Application of the spectral stochastic finite element method for performance prediction of composite structures. Compos Struct 2007; 78(3): 447-56.

[4] Kim HK, Inoue J. A spectral stochastic element free Galerkin method for the problems with random material parameter. Int $\mathrm{J}$ Numer Methods Eng 2004; 61(11): 1957-75.

[5] Sheu GY. Prediction of probabilistic settlements via spectral stochastic meshless local Petrov-Galerkin method. Comput Geotech 2011; 38(4): 407-15.

[6] Atlrui SN, Zhu T. A new meshless local Petrov-Galerkin (MLPG) approach in computational mechanics. Comput Mech 1988; 22(2): 117-27.

[7] Liu GR, Gu YT. A meshfree method: meshfree weak-strong (MWS) form method, for 2-D solids. Comput Mech 2004; 33(1): 214.

[8] Kaminski, M. Generalized perturbation-based stochastic finite element in elastostatics. Comput Struct 2007; 85(10): 586-94.

[9] Sudret B, Kiureghian AD. Stochastic finite elements and reliability: a state-of-the-art report. Technical Report 2000, UCB/SEMM2000/08. Berkeley: University of California 2000.

[10] Liu GR. Meshfree methods: moving beyond the finite element method. Boca Raton, USA: CRC press 2002.

[11] Rahman S, Rao BN. A perturbation method for stochastic meshless analysis in elastostatics. Int J Numer Methods Eng 2001; 50(8): 1969-91. 